\title{
Theory of Melting and the Optical Properties of Gold/DNA Nanocomposites
}

\author{
Sung Yong Park and D. Stroud \\ Department of Physics, The Ohio State University, Columbus, Ohio 43210
}

(October 30, 2018)

\begin{abstract}
We describe a simple model for the melting and optical properties of a DNA/gold nanoparticle aggregate. The optical properties at fixed wavelength change dramatically at the melting transition, which is found to be higher and narrower in temperature for larger particles, and much sharper than that of an isolated DNA link. All these features are in agreement with available experiments. The aggregate is modeled as a cluster of gold nanoparticles on a periodic lattice connected by DNA bonds, and the extinction coefficient is computed using the discrete dipole approximation. Melting takes place as an increasing number of these bonds break with increasing temperature. The melting temperature corresponds approximately to the bond percolation threshold.

PACS numbers: 61.43.Hv, 78.67.-n, 82.60.Qr, 87.15.-v
\end{abstract}

The optical properties of metallic nanoparticles have been investigated intensively over the last decades $[1,2]$. Recently, this investigation has expanded to include so-called functional metallic nanoparticles [3]. Among these, there is a particular interest in DNA-modified gold nanoparticles. Such particles can form complex aggregates which may melt at high temperatures. The particles and their aggregates may have a variety of applications, e. g., in biological detection, which may be possible by using the optical and electrical sensitivity of the aggregates [4-6]. Numerical model calculations of the optical properties of DNA modified gold nanoparticle aggregates show general agreement with experiments, including such features as the following: (i) for isolated gold nanoparticles in suspension, there is a strong "surface plasmon" absorption in the visible; and (ii) this absorption maximum broadens and red-shifts when the cluster radius becomes comparable to the wavelength $[7,8]$. However, some important physical details have not been explained. For example, in the gold/DNA nanoparticle system, the melting transition of a gold nanoparticle aggregate has a much narrower temperature width and occurs at a high temperature than that of a single DNA link [5,9]. Also, the dependence of aggregate melting temperature on particle size has not yet been explained [10].

In this Letter, we model this novel "melting" transition of a gold/DNA nanoparticle aggregate. Our model accounts for most experimentally observed features, including (i) the small temperature width of the aggregate melting transition, compared to that of a single DNA link; (ii) the particle-size-dependence of the melting temperature $T_{m}$; and (iii) the temperature-dependence of the optical extinction coefficient $C_{\text {ext }}(\lambda, T)$ at wavelength $\lambda$ and temperature $T$.

We first describe our model for the low-temperature morphology of the aggregate. In the simplest version of our model, the low-T cluster is taken simply as a collection of identical gold nanoparticles (each of radius $a$ ) which fill the sites of a simple cubic lattice of lattice constant $d(d>2 a)$. The cluster is assumed to be a cube of edge $L$, containing $N_{\text {par }}=(L / d)^{3}$ gold nanoparticles. We have also investigated the melting and the optical properties assuming that the low- $\mathrm{T}$ cluster is a fractal aggregate.

To describe the melting of this aggregate, we assume that each nanoparticle carries exactly $N_{s}$ single DNA strands. (This choice still retains the essential features of the melting.) The cluster exists at low $T$ because a chemical reaction converts two single strands $S$ into a double strand $D$. In this Letter, we adopt a simplified two-state model for this reaction $[11,12]$, described by the relation

$$
S+S \rightleftharpoons D \text {. }
$$

For short DNA strands (12-14 base pairs), this model describes melting well [13]. Each gold nanoparticle has $z$ nearest neighbors $(z=6$ for a simple cubic lattice). Hence, there are $N_{\mathrm{par}} z / 2$ bonds joining adjacent nanoparticles. We assume that a (temperaturedependent) fraction $p(T)$ of the single DNA strands form double strands by the reaction (1). In order to calculate the fraction $p_{\text {eff }}(T)$ of bonds which contain at least one double strand, we adopt the following model. First, we assume that exactly $N_{s} / z$ of the single strands on a given nanoparticle are available to bond with any one of its $\mathrm{z}$ nearest neighbors. Thus, we assume that the maximum number of links that could be formed between any two particles is $N_{s} / z$. The probability that no link is formed is then taken to be

$$
1-p_{\text {eff }}(T)=[1-p(T)]^{N_{s} / z} .
$$

The criterion for the melting temperature $T_{c}$ is that $p_{\text {eff }}\left(T_{c}\right)=p_{c}, p_{c}$ being the bond percolation threshold [14] for the lattice considered, at which an infinite connected path of double DNA strands first forms. (For example, $p_{c} \sim 0.25$ on a very large simple cubic lattice.) Thus, at percolation, 


$$
\left[1-p\left(T_{c}\right)\right]^{N_{s} / z}=1-p_{c} .
$$

This equation implicitly determines $T_{c}$ in terms of $z, p_{c}$, and $N_{s}[15]$.

$p(T)$ itself is determined by a chemical equilibrium between the single-strand and double-strand DNA molecules, which are attached to the gold nanoparticles. The chemical equilibrium condition corresponding to (1) is

$$
\frac{[1-p(T)]^{2}}{p(T)}=\frac{K(T)}{C_{T}} \equiv K^{\prime}(T),
$$

where $K(T)$ is a suitable chemical equilibrium constant, and $C_{T}$ is the molar concentration of single DNA strands in the sample (in our model all are attached to the nanoparticles). Since $0<p(T)<1$, the physical solution to eq. (4) is $p(T)=1+\frac{1}{2}\left(K^{\prime}-\sqrt{K^{\prime, 2}+4 K^{\prime}}\right)$.

Note that, according to eq. (3), $p\left(T_{c}\right)$ decreases with increasing $N_{s}$ and, hence, with increasing particle radius. Since $p(T)$ decreases monotonically with $T, T_{c}$ should thus be an increasing function of $a$, as reported in experiments [10].

In Fig. 1, we plot $\mathrm{p}_{\text {eff }}(\mathrm{T})$ for several $a$. We assume $N_{s} \propto a^{2}$, set $z=6$ and use the experimental result that $N_{s}=160$ when $a=8 \mathrm{~nm}$ [16]. We have also assumed the simple van't Hoff behavior $K(T)=$ $\exp \left[-\Delta G / k_{B} T\right]$, with a Gibbs free energy of formation $\Delta G(T)=c_{1}\left(T-T_{M}\right)+c_{3}\left(T-T_{M}\right)^{3}$, choosing the values of $c_{1}, c_{3}$, and $T_{M}$ to be consistent with experiments on these DNA molecules.

To calculate the $T$-dependent optical properties, we have considered the melting of two slightly different lowtemperature aggregates. In the first, we assume that, at low $T$, the aggregate consists of a simple cubic collection of $N_{\text {par }}=(L / d)^{3}$ gold nanoparticles on a simple cubic lattice, as described above, with all bonds occupied by DNA double-strand links. To generate a specific sample with a given $p_{\text {eff }}(T)$, we randomly remove links with probability $1-p_{\text {eff }}(T)$, then identify the separate clusters, using a simple computer algorithm [14]. We neglect gravitational forces, which may be important in some experimental circumstances. If the aggregate consists of two or more clusters, we simply place these aggregates in random positions and orientations within a larger bounding box (usually of edge 100d), taking care that the individual clusters do not overlap. The resulting geometry is shown schematically in Fig. 2. We have also carried out the same procedure to simulate the melting of a sample formed by reaction-limited cluster-cluster aggregation (RLCA) [17]. A typical RLCA cluster is shown in Fig. 2 (d), and represents a possible fractal aggregate which might be produced by certain random growth processes at low $T$ [18].

We calculate the optical properties of this sample using the Discrete Dipole Approximation (DDA) $[19,20]$.
The sample is modeled as a collection of separate aggregates whose extinction coefficients are computed individually, then added. Each aggregate consists of many identical nanoparticles, which have complex frequencydependent dielectric constant $\epsilon(\omega)$, and polarizability $\alpha(\omega)$ related to $\epsilon(\omega)$ by the Clausius-Mossotti equation [21] $\epsilon(\omega)=1+4 \pi n \alpha /[1-(4 \pi n / 3) \alpha]$, where $n=(d / L)^{3}$. The resulting expressions for the induced dipole moment $\mathbf{p}_{i}$ of the $i^{t h}$ sphere, and the corresponding expression for the extinction coefficient $C_{\text {ext }}(k)$ at wave number $k$, are given in Ref. [7].

In our case, each cluster consists of a number of DNAlinked individual gold nanoparticles. In our calculations, we do not include the optical properties of the DNA molecules, since these absorb primarily in the ultraviolet [8]. We use tabulated values of the gold complex index of refraction $[22,23]$, then calculate $C_{e x t}$ for each cluster using the DDA. To improve the statistics, we average $C_{e x t}$ for each cluster over possible orientations. We then sum the averaged extinction coefficients of all the individual clusters to get the total extinction coefficient of the suspension. This method is justified when the suspension is dilute.

In Fig. 3, we show $C_{\text {ext }}(\lambda, T)$ versus $\lambda$ for the goldDNA cluster at several values of $\mathrm{p}_{\mathrm{eff}}(\mathrm{T})$, assuming $a=20$ nm. $\mathrm{p}_{\text {eff }}=0$ represents a dispersion of $N_{\text {par }}=(L / d)^{3}$ individual nanoparticles, while $p_{\text {eff }}=1$ represents a simple cubic lattice of connected nanoparticles. The calculated $C_{\text {ext }}(\lambda, T)$ at each $\lambda$ changes strikingly for $\mathrm{p}_{\text {eff }}(\mathrm{T}) \sim \mathrm{p}_{c}$. For small $p_{\text {eff }}$, there is a clear extinction peak near 520 $\mathrm{nm}$. This peak corresponds to the wavelength of the surface plasmon resonance (SPR) in individual Au nanoparticles. As $p_{\text {eff }}$ increase, this peak first red-shifts, then greatly broadens, as the aggregate melts. The calculated peak is, however, shifted much more and is much broader than experiment. However, as shown in inset, if we assume that the low- $T$ aggregate is an RLCA fractal, the calculated peak shift is consistent with experiment [18].

In Fig. 4, we show $C_{\text {ext }}(\lambda, T)$ at fixed $\lambda=520 \mathrm{~nm}$ (close to the isolated-particle SPR), versus $T$, for several particle sizes, assuming that the low- $\mathrm{T}$ aggregate is a simple cubic cluster with $N=1000$ particles, as shown in Fig. 2. For each $a$, the extinction increases sharply at a characteristic $T$, corresponding to the melting of the aggregate for that $a$; at this $T$, the absorption due to the SPR increases sharply.

In the inset of Fig. 4, we compare $C_{e x t}(\lambda, T)$ for a regular and an RLCA cluster of particles of $20 \mathrm{~nm}$ radius at $\lambda=520 \mathrm{~nm}$, both for $N=1000$ particles. Although the RLCA cluster has a slightly broader melting transition, as manifested in $C_{\text {ext }}(\lambda, T)$, than does the regular lattice, both sets of data show a much sharper melting transition than that of a single DNA link. Also, although our normalized $C_{\text {ext }}(\lambda, T)$ is calculated for the aggregates at $520 \mathrm{~nm}$, we expect similar behavior at $260 \mathrm{~nm}$. (We have not carried out calculations at this $\lambda$ mainly because 
we have not included the DNA absorption properties.) In any case, the experimental melting curves at 260 and 520 nm are very similar [10].

Our calculated extinction coefficients are strikingly similar to recent experimental results $[5,8,10]$. In particular, both experiment and calculation give a sharp increase in $C_{\text {ext }}(\lambda, T)$ at fixed $\lambda$, as $T$ increases past a critical temperature, which we interpret as the melting temperature $T_{m}$. We also find, in agreement with experiment $[5,8]$, that melting occurs over a much narrower range of $T$ in the aggregate than for a single bond, and that the melting occurs at higher $T$ for larger particles [10]. The crucial point is that $p_{\text {eff }}(T)$ is a much sharper function of $T$ than is $p(T)$, and this feature would not be affected by slight changes in the model (such as considering a body-centered-cubic rather than a simple cubic cluster). In the present model, the melting transition is in the universality class of bond percolation. This universality class might change because of the constant dissolving and reforming of the bonds of the aggregate (a possibility omitted from our model). This possibility should be studied further theoretically and experimentally. However, the optical properties may well be robust, in the sense that $C_{\text {ext }}(T)$ would be little affected even if this feature were included, since the key ingredient is the multiple links per bond as described above.

In summary, we have developed a simple model for the melting of aggregates of gold nanoparticles and DNA, and have calculated the T-dependent optical properties of these melting aggregates, using the DDA. We find that, at fixed $\lambda$, melting is accompanied by dramatic changes in the extinction coefficient $C_{\text {ext }}(\lambda)$. These calculated changes occur over a much narrower temperature range than that over which interparticle links themselves melt, the temperature width is smaller for particles with larger radius, and the melting occurs at higher temperatures for the larger nanoparticles than for the smaller ones. All these effects are in good agreement with experiment $[5,8,10]$. It would be of great value if the predicted $T$ dependent structure of the clusters could be probed experimentally, e. g. by static light scattering, as has been done in other contexts [24]. Finally, we mention that the present model can be applied to other similar nanocomposites [25].

This work has been supported by grant NSF DMR0104987, by the U. S./Israel Binational Science Foundation, and by an Ohio State University Postdoctoral Fellowship awarded to S. Y. Park. We thank D. J. Bergman, C.-H. Kiang, A. A. Lazarides, and D. R. Nelson for valuable conversations.
[1] C. F. Bohren and D. R. Huffman, Absorption and Scattering of Light by Small Particles, (John Wiley \& Sons, New York, 1983).

[2] U. Kreibig and M. Vollmer, Optical Properties of Metal Clusters, (Springer-Verlag, Berlin, 1995).

[3] C. Sanchez, G. J. de A.A. Soler-Illia, F. Ribot, T. Lalot, C. R. Mayer, and V. Cabuil, Chem. Mater. 13, 3061 (2001).

[4] C. A. Mirkin, R. L. Letsinger, R. C. Mucic, J. J. Storhoff, Nature 382, 607 (1996).

[5] R. Elghanian, J. J. Storhoff, R. C. Mucic, R. L. Letsinger, and C. A. Mirkin, Science 277, 1078 (1997); J. J. Storhoff, R. Elghanian, R. C. Mucic, C. A. Mirkin, R. L. Letsinger, J. Am. Chem. Soc. 120, 1959 (1998).

[6] S.-J. Park, T. A. Taton, and C. A. Mirkin, Science 295, 1503 (2002).

[7] A. A. Lazarides and G. C. Schatz, J. Phys. Chem. B 104, 460 (2000), and references cited therein.

[8] J. J. Storhoff, A. A. Lazarides, R. C. Mucic, C. A. Mirkin, R. L. Letsinger, and G. C. Schatz, J. Am. Chem. Soc. 122, 4640 (2000).

[9] K. Drukker, G. Wu, and G. C. Schatz, J. Chem. Phys. 114, 579 (2001).

[10] C.-H. Kiang and R. Ramos, Los Alamos preprint physics/0111002 (November, 2001).

[11] V. A. Bloomfield, D. M. Crothers, and I. Tinoco, Jr., Nucleic Acids (University Science Books, Sausalito, California, 2000).

[12] In actuality, there is a linker molecule which emerges from solution to connect two DNA single strands, each on a different nanoparticle. Even in this case, however, the melting condition can be reduced to that used here, without loss of accuracy, as will be shown elsewhere. Also, there are two chemically distinct DNA single strands (denoted $\mathrm{A}$ and $\mathrm{B}$ ); each gold particle has either all A or all B single strands attached, and linker molecule can only attach A and B single strands. The existence of two species can be disregarded by symmetry when the the concentrations of the two species in solution are the same; experiments have been carried out only under this condition.

[13] H. Werntges, G. Steger, D. Riesner, and H.-J. Fritz, Nucleic Acids Res. 14, 3773 (1986).

[14] See, e. g., D. Stauffer and A. Aharony, Introduction to Percolation Theory, 2nd Edition (Taylor and Francis, London,1992).

[15] We implicitly assume that the specific bonds which are occupied at temperature $T$ are time-independent. In reality, these links slowly break up and reform in time. The effects of this assumption are discussed further below.

[16] L.M. Demers, C.A. Mirkin, R.C. Mucic, R. A. Reynolds, III, R.L. Letsinger, R. Elghanian, and G. Viswanadham, Anal. Chem. 72, 5535 (2000).

[17] For the actual computer algorithm to produce the RLCA clusters, see, e. g., W. D. Brown and R. C. Ball, J. Phys. A 18, L517 (1985), or P. Meakin, Adv. Colloid Interface Sci. 28, 249 (1988).

[18] S. Y. Park and D. Stroud, (unpublished).

[19] E. M. Purcell and C. R. Pennypacker, Astrophys. J. 186, 705 (1973).

[20] B. T. Draine and P. J. Flatau, J. Opt. Soc. Am. A 1491, 11 (1994). 
[21] See, e. g., E. M. Purcell, Electricity and Magnetism, Berkeley Physics Course, Vol. 2 (McGraw-Hill, New York, 1965).

[22] D. W. Lynch and W. R. Hunter, in Handbook of Optical Constants of Solids, E. D. Palik, ed. (Academic, New York, 1985), p. 294.

[23] P. B. Johnson and R. W. Christy, Phys. Rev. B 6, 4370 (1972).

[24] M. Y. Lin, H. M. Lindsay, D. A. Weitz, R. C. Ball, R. Klein, and P. Meakin, Phys. Rev. A 41, 2005 (1990).

[25] S. Mann, W. Shenton, M. Li, S. Connolly, and D. Fitzmaurice, Adv. Mater. 12, 147 (2000); A. K. Boal, F. Ilhan, J. E. DeRouchey, T. Thurn-Albrecht, T. P. Russell, and V. M. Rotello, Nature 404, 746 (2000).

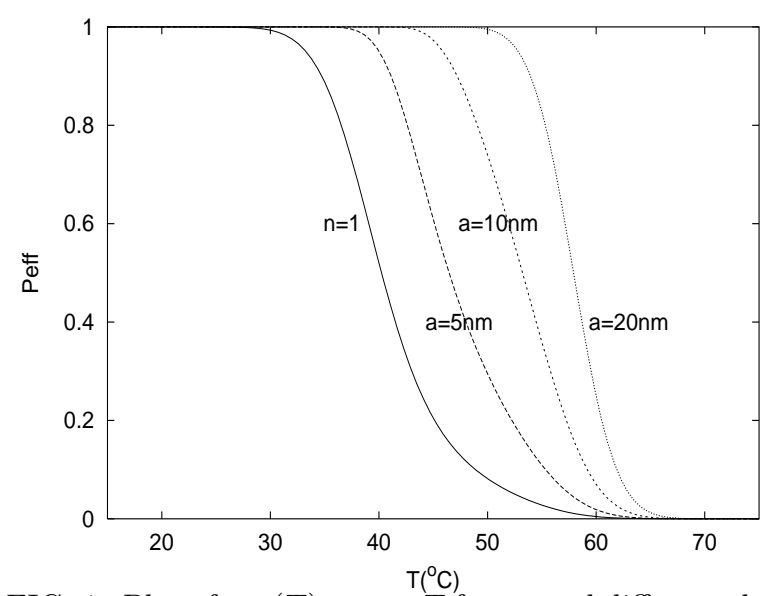

FIG. 1. Plot of $p_{\text {eff }}(T)$ versus $T$ for several different choices of particle radius $a$, as indicated. Also plotted is $\mathrm{p}(\mathrm{T})$, the probability that a given DNA strand is part of a double strand at $T$.

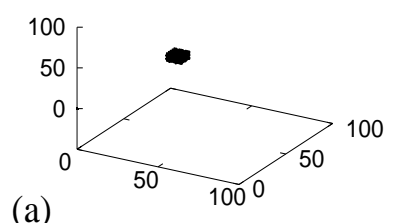

(a)
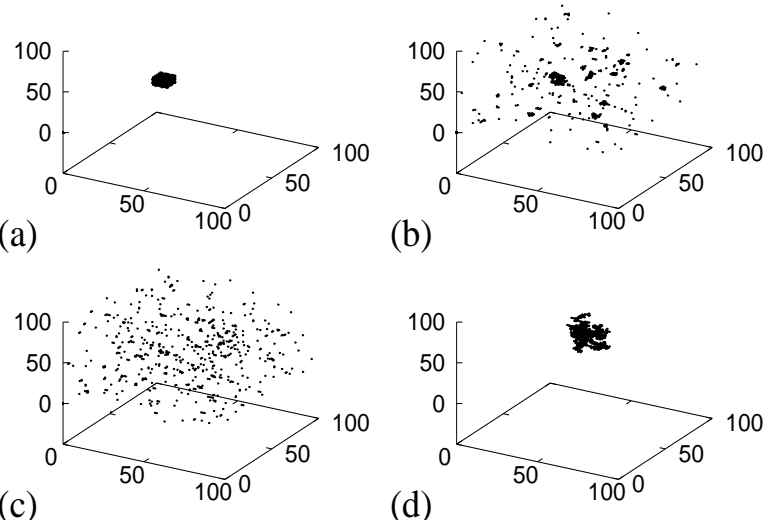

FIG. 2. Schematic of the melting of a gold-DNA cluster, for two different models discussed in the present paper. In the first model, (a) at low $T$ the cluster is described as an $L \times L \times L$ simple cubic aggregate of lattice constant $d$ [here $L / d=10]$. (b) As the temperature $T$ increases, some of the bonds break. The fraction of bonds present is $p_{\text {eff }}(T)$. In (b), $p_{\text {eff }}=0.5>p_{c}(L)$, where $p_{c}$ is the weakly $L$-dependent percolation threshold. A percolation cluster of linear dimension $\sim L$ still exists. At a still higher $T$ [shown in (c)], $p_{\text {eff }}(T)=0.2<p_{c}(L)$, and only small clusters remain. In our calculation, the clusters are positioned and oriented at random in the bounding box, taken as a cube of edge $100 d$. (d) Alternate model for low- $T$ sample $\left(p_{\text {eff }}(T)=1\right)$ : fractal cluster formed by reaction-limited cluster-cluster aggregation [RLCA], with fractal dimension $d_{f} \sim 2.1$.

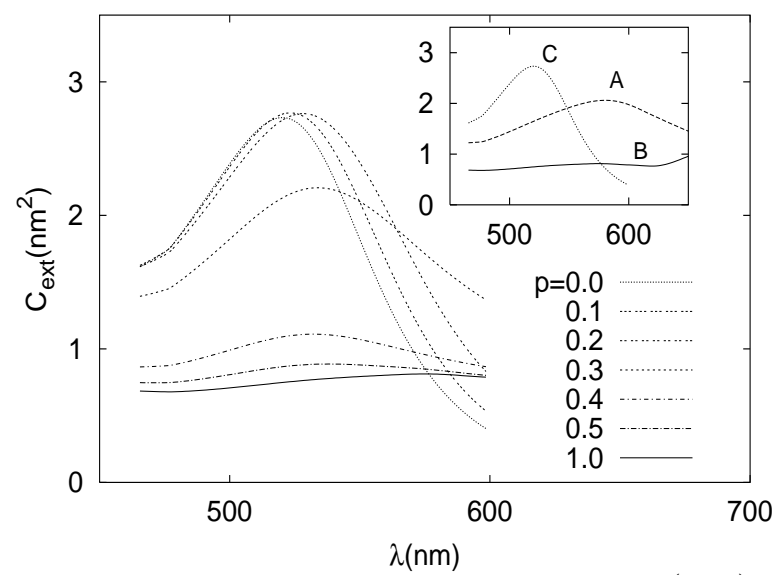

FIG. 3. Calculated extinction coefficient $C_{\text {ext }}(\lambda, T)$ as a function of wavelength $\lambda$, plotted for several values of $p_{\text {eff }}$, particle radius $20 \mathrm{~nm}$, as indicated in the legend. $p_{\text {eff }}=1$ corresponds to 1000 gold nanoparticles on a simple cubic lattice of edge $L=480 \mathrm{~nm}$. The sample is that shown schematically in Fig. 2 (a) - (c). Inset: $C_{\text {ext }}(\lambda, T)$ for the RLCA aggregate at $p_{\text {eff }}=1$ (curve A), as well as for simple cubic aggregate at $p_{\text {eff }}=1$ (curve $\mathrm{B}$ ), and for individual gold nanoparticles $p_{\text {eff }}=0 .($ curve $\mathrm{C})$.

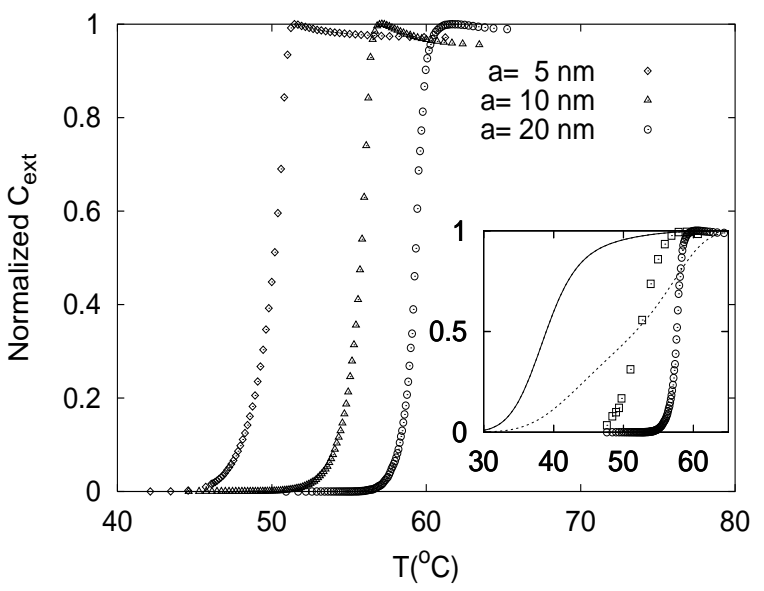


FIG. 4. Normalized extinction coefficient $C_{\text {ext }}(\lambda, T)$ for $\lambda=520 \mathrm{~nm}$, plotted versus $T$ for several particle radii $a$, assuming that the low- $T$ aggregate is an $N=1000$ simple cubic cluster, as shown in Fig. 2. Inset: Normalized extinction coefficient $C_{\text {ext }}(\lambda, T)$, versus $T$ for $\lambda=520 \mathrm{~nm}$, plotted for a 1000-particle gold/DNA aggregate assuming that the low-temperature sample is a simple cubic cluster (open circles) or an RLCA cluster (open squares). The solid curve is a plot of $1-p(T)$ for a single DNA duplex with the same concentration $C_{T}$ as the above two curves. The dotted curve represents $1-p(T)$ for a single DNA duplex but with a much higher $C_{T}$ than for the other curves of the inset. The plots of $1-p(T)$ for a single link closely resemble $C_{\text {ext }}(\lambda, T)$ as measured at $\lambda=260 \mathrm{~nm}$ for a single duplex[11]. 